\title{
Ramzan for Patients with Chronic Liver Disease
}

\author{
Zain Majid* \\ Department of Hepato-gastroenterology, Sindh Institute of Urology and Transplantation, Pakistan
}

*Corresponding author: Zain Majid, Department of Hepato-gastroenterology, Sindh Institute of Urology and Transplantation, SIUT, Karachi, Pakistan.

Received Date: April 14, 2021

Published Date: May 07, 2021

\section{Editorial}

With the holy month of Ramzan fast approaching us, Muslim's from all around the world would be engaged in various religious activities including fasting. Ramzan is the 9th month of the Islamic calendar and during which fasting is obligatory for all muslims. During a fast a Muslim not only abstains themselves from food and water but also from smoking and oral medications [1]. A typical fast last around 12-18 hours [2] At present the impact of fasting on patients with chronic liver disease regardless of its etiologic is scarce. Here we wish to highlight the effects of fasting in the month of ramzan and the precautionary measures that need to be taken in this month in patients diagnosed with chronic liver disease (regardless of its etiology). Cirrhotics are particularly at risk of protein energy malnutrition (PEM), upper gastrointestinal bleed, worsening of their liver functions along with encephalopathy and discontinuation of their medications during fasting [3]. Along with this, fasting in the tropics or during the summer season may place these patients at risk of renal dysfunction [3]. Generally, it is recommended that patients having Child Pugh score C (CPS) cirrhosis should avoid fasting [3].

A study done in Egypt on Cirrhotics during the month of Ramzan showed an increase in liver congestive index from fasting to post prandial state, this increases the portal blood flow and the risk of bleeding [3]. Cirrhotics living in the northern hemisphere face with a even more challenging situation when Ramzan falls in the summer months as the duration of their fast is longer and both meals of the day are taken close together [3]. Malnutrition is seen
$25 \%$ of patients have Child class A cirrhosis while is noted in $80 \%$ of those with Child class C [3]. Another study showed that respiratory quotient (RQ) after an overnight fast is lower in cirrhotic patients as compared to normal person, reflecting catabolism and an accelerated fat oxidation [4]. Another research showed the positive effects of alternate day fasting in patients with Non-alcoholic fatty liver disease (NAFLD) and led to weight loss, improvement in the dyslipidaemia within 12 weeks [5].

\section{Conclusion}

Cirrhotics with CPS C should avoid fasting since this can lead to clinical deterioration and worsening of their liver function tests. While those with CPS A, B can fast provide they have good medical along with nutritional care.

\section{Acknowledgment}

None.

\section{Conflict of Interest}

No conflict of interest.

\section{References}

1. Sakr AH (1975) Fasting in Islam. J Am Diet Assoc 67(1): 17-21.

2. Elnadry MH, Nigm IA, Abdel Aziz IM, Elshafee AM, Elazhary SS, et al. (2011) Effect of Ramadan fasting on Muslim patients with chronic liver diseases. J Egypt Soc Parasitol 41(2): 337-346.

3. Mohamed S, Emara M, Hussien H, Elsadek H (2016) Changes in portal blood flow and liver functions in cirrhotics during Ramadan fasting in the summer; a pilot study. Gastroenterol Hepatol Bed Bench 9(3): 180188. 
4. Nakaya Y, Harada N, Kakui S, Okada K, Takahashi A, et al. (2002) Severe catabolic state after prolonged fasting in cirrhotic patients: effect of oral branched-chain amino-acid-enriched nutrient mixture. J Gastroenterol 37(7): 531-536.
5. Hua Cai, Yue-Lan Qin, Ze-Ya Shi, Jin-Hui Chen, Min-Jie Zeng, et al. (2019) Effects of alternate-day fasting on body weight and dyslipidemia in patients with non-alcoholic fatty liver disease: a randomised controlled trial. BMC Gastroenterol 19(1): 219. 